\title{
Electric-Jet Assisted Layer-by-Layer Deposition of Gold Nanoparticles to Prepare Conducting Tracks
}

\author{
S. R. Samarasinghe ${ }^{1}$, Isabel Pastoriza-Santos ${ }^{3}$, M. J. Edirisinghe ${ }^{1^{*}}$, M. J. Reece ${ }^{2}$, \\ Luis M. Liz-Marzán ${ }^{3}$, M. R. Nangrejo ${ }^{1}$, Z. Ahmad \\ ${ }^{1}$ Department of Mechanical Engineering, University College of London, Torrington Place, London. WC1E 7JE, UK; \\ m.edirisinghe@ucl.ac.uk \\ ${ }^{2}$ Centre for Materials Research, Queen Mary, University of London, Mile End Road, London. E1 4NS, UK \\ ${ }^{3}$ Department of Physical Chemistry, University of Vigo, Vigo 36310, Spain
}

Received 18 July 2009; revised 30 July 2009; accepted 3 August 2009.

\begin{abstract}
A suspension of $15 \mathrm{~nm}$ diameter gold nanoparticles has been deposited along a line on a silicon substrate with the assistance of a jet generated in an electric field. In order to control the evaporation of the solvent used to suspend the gold nanoparticles, a heating device was used to change the substrate temperature. Layer-bylayer deposition enabled the direct writing of gold tracks having an electrical resistivity of $\mathbf{1 . 8}$ $\times 10^{-7} \Omega \mathrm{m}$, only about an order of magnitude above the electrical resistivity of bulk gold.
\end{abstract}

Keywords: Gold; Electrohydrodynamic; Jet; Direct Write; Track; Electrical Conductivity

\section{INTRODUCTION}

The forming of fine metallic patterns from colloids and suspensions is gaining tremendous interest because it is a potential fabrication route for the next generation electronic devices. Techniques, such as electron-beam lithography and photo-lithography are the most popular patterning techniques, and are at the heart of modern day microfabrication, nanotechnology and molecular electronics. Lithography techniques require a mask or resistive film to pattern microstructures on substrates and thereafter harsh chemical etching is needed to produce the final pattern. This makes them unsuitable for patterning nanoparticles or molecules with organic or biological functionalities, since it impairs the organic molecules and biological entities [1]. In addition, these lithographic techniques are not only time consuming but also quite complicated. For these reasons, the development of convenient and fast processing techniques to fabricate conductive lines has attracted more attention in recent years [2].
Recently developed techniques, for example, microcontact printing, also require an elastomer stamp for patterning, which can deform due to its elastomeric nature, resulting in distorted patterns [3]. Dip-pen lithography is also a recently developed patterning technique, which allows direct transporting and patterning of particles and molecules at nanometer scale $(30-100 \mathrm{~nm})$ onto a substrate from the tip of an atomic force microscope. However, this technique can usually convey only a small amount of materials, since the transfer efficiency is relatively low [1].

Direct write technologies have been explored recently for fabricating fine patterns whose line widths range from the meso to the nanoscale. The term direct write refers to any technique or process that is capable of depositing, dispensing or processing different types of materials on various surfaces following a preset pattern or layout. The main advantages of the direct write approach is that patterns or structures can be obtained without the use of moulds or pre-fabrication processes, masks, and liquids for etching. Direct write technologies are therefore low cost, high speed, non-contact and environmentally friendly processes $[4,5]$.

As a non-contact patterning technique, ink-jet printing (IJP) has been used in the last decade for a number of new applications, such as the fabrication of organic light-emitting diodes, transistors and integrated circuits, conducting polymer devices, structural polymers, ceramics and biomolecular arrays [6]. Table 1 shows recent publications on fabricating conducting tracks by ink-jet printing and the concentration and resistivities achieved by other researchers. The metal concentration used in most of the studies varies from $20-48 \mathrm{wt} . \%$.

Printing using a jet generated in an electric field is an emerging direct write patterning technology. In this method, the medium is made to jet and disperse into fine droplets, which are deposited on a substrate using computer control to form a pre-designed pattern. Deposition 
Table 1. Summary of recent works on fabricating conducting tracks by IJP.

\begin{tabular}{ccccccc}
\hline Metal & $\begin{array}{c}\text { Particle } \\
\text { Size }(\mathbf{n m})\end{array}$ & Width $(\boldsymbol{\mu m})$ & $\begin{array}{c}\text { Conc. } \\
\mathbf{( w t . \% )}\end{array}$ & $\begin{array}{c}\text { Curing } \\
\text { Condition }\end{array}$ & $\begin{array}{c}\text { Resistivity } \\
(\mathbf{\Omega m})\end{array}$ & Ref. \\
\hline $\mathrm{Ag}$ & $10-20$ & 60 & 34.5 & Electrical & $2.7 \times 10^{-8}$ & {$[10]$} \\
$\mathrm{Ag}$ & 21 & 90 & 20 & $150{ }^{\circ} \mathrm{C}$ & $3.2 \times 10^{-8}$ & {$[11]$} \\
$\mathrm{Ag}$ & $1-10$ & 125 & 30 & $300{ }^{\circ} \mathrm{C}$ & $3.5 \times 10^{-7}$ & {$[12]$} \\
$\mathrm{Ag}$ & 20 & 65 & 20 & $300{ }^{\circ} \mathrm{C}$ & $3.5 \times 10^{-8}$ & {$[13]$} \\
$\mathrm{Ag}$ & - & 100 & 16 & $150{ }^{\circ} \mathrm{C}$ & $4.8 \times 10^{-8}$ & {$[14]$} \\
$\mathrm{Ag}$ & - & 750 & 48 & $300{ }^{\circ} \mathrm{C}$ & $1.5 \times 10^{-7}$ & {$[15]$} \\
$\mathrm{Ag}$ & $10-50$ & 130 & 25 & $260{ }^{\circ} \mathrm{C}$ & $1.6 \times 10^{-7}$ & {$[2]$} \\
$\mathrm{Ag}$ & $5-10$ & 160 & 60 & Microwave & $1.6 \times 10^{-7}$ & {$[16]$} \\
$\mathrm{Ag}$ & $5-7$ & 1500 & 10 & $320{ }^{\circ} \mathrm{C}$ & $1.1 \times 10^{-6}$ & {$[6]$} \\
$\mathrm{Au}$ & $2-4$ & 17 & 30 & Laser & $1.4 \times 10^{-7}$ & {$[17]$} \\
$\mathrm{Au}$ & $2-5$ & 20 & 30 & Laser & $1.4 \times 10^{-7}$ & {$[18]$} \\
$\mathrm{Au}$ & - & 360 & 31 & $500{ }^{\circ} \mathrm{C}$ & $2.7 \times 10^{-7}$ & {$[19]$} \\
$\mathrm{Au}$ & $2-4$ & 125 & 34 & Laser & $6.2 \times 10^{-8}$ & {$[20]$} \\
$\mathrm{Cu}$ & $40-50$ & 65 & 20 & $325{ }^{\circ} \mathrm{C}$ & $1.72 \times 10^{-7}$ & {$[21]$} \\
\hline
\end{tabular}

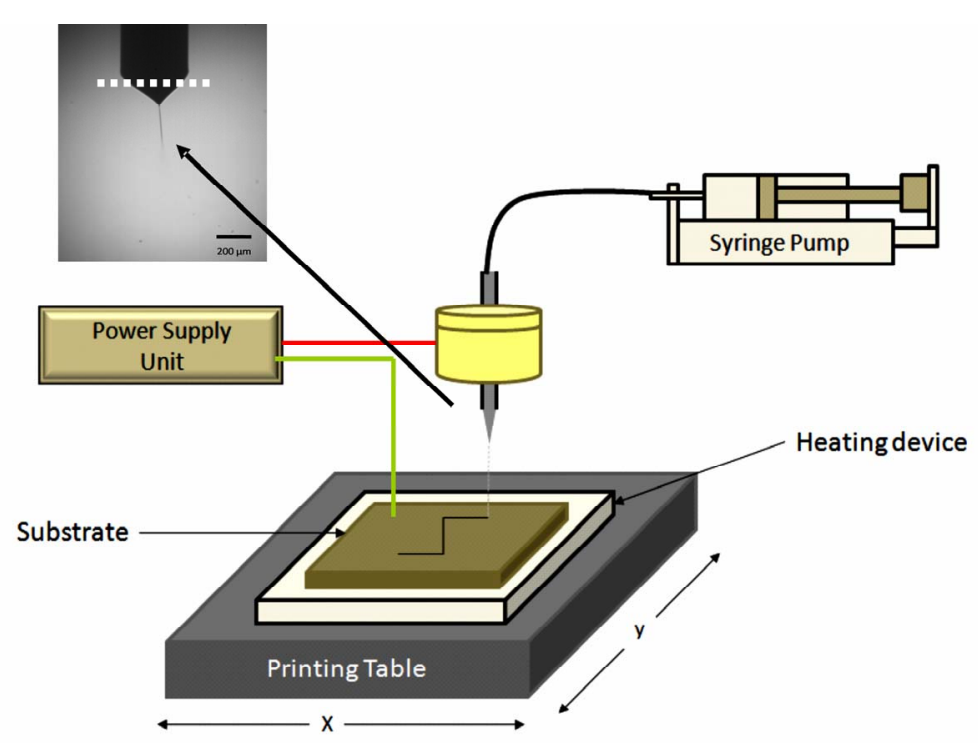

Figure 1. Schematic diagram of the electrohydrodynamic jet printing process.

of materials using electric field assisted jet printing offers some advantages over other non-contact printing techniques. Firstly, by careful tailoring of the physical properties of the medium and by suitably fixing its flow rate and the applied voltage controlling the electric field it is possible to produce very fine droplets in the range of $40 \mathrm{~nm}-1.8 \mu \mathrm{m}[7,8]$. Second, the diameter of the capillaries (needles, nozzles) used in this method is much coarser $(>100 \mu \mathrm{m}$ inner diameter) than the capillaries used in ink-jet printing (usually around $20-60 \mu \mathrm{m}$ ). The use of larger capillaries reduces the possibilities of blockages and allows viscous suspension containing high volume loading (above $30 \mathrm{vol} . \%$ ) of solid particles to be processed [9].

Electric-field assisted jet printing has been used to produce conducting tracks from silver nanoparticles $[22,23]$, the silver concentrations used in their experiments varied from $20-30$ wt. \%. In contrast, in this 
paper, a low concentration gold suspension ( $\sim 0.1 \mathrm{wt} . \%)$ containing $15 \mathrm{~nm}$ diameter particles was used to deposit conducting tracks layer-by-layer with the aid of a jet generated in an electric field.

\section{EXPERIMENTAL DETAILS}

\subsection{Preparation of Gold Suspension}

Tetrachloroauric acid and trisodium citrate were purchased from Aldrich. Poly (vinylpyrrolidone) (PVP, molecular weight 10,000) was supplied by Fluka. All of the chemicals were used as received and milli-Q water was used to make up all solutions $(\mathrm{R}>18.2 \mathrm{M} \Omega \mathrm{cm})$. Gold nanoparticles with an average diameter of $15 \mathrm{~nm}$ and $10 \%$ polydispersity were synthesized according to the standard sodium citrate reduction method [24] by boiling $5 \times 10^{-4}$ $\mathrm{M} \mathrm{HAuCl}_{4}$ in the presence of $1.6 \times 10^{-3} \mathrm{M}$ sodium citrate for 900s. After cooling down, the particles were transferred into ethanol upon functionalization with PVP [25]. Briefly, an amount of PVP sufficient to coat the particles with 60 PVP monomers per $\mathrm{nm}^{2}$ was dissolved by ultrasonication for $900 \mathrm{~s}$ in water and added to the gold colloid. The polymer was allowed to adsorb to the gold particles overnight while stirring. The particles were subsequently centrifuged (3500 r.p.m.) to remove the unbound PVP and redispersed in ethanol.

\subsection{Electric-field Assisted Jetting and Deposition}

The apparatus (Figure 1) consists of a programmable syringe pump (Harvard Apparatus Ltd., Edenbridge, UK) supplying the gold suspension to the stainless steel nozzle (internal diameter of $\sim 200 \mu \mathrm{m}$ and external diameter of $\sim 400 \mu \mathrm{m}$ ) held in epoxy resin. The electrical power supply unit consisted of a high voltage power supply (Glassman Europe Ltd., Tadley, UK) capable of supplying up to $30 \mathrm{kV}$ between the electrodes.

A custom built printing device was used to pattern microstructures on substrates. It consists of a stepper motor driven 2D system and the $\mathrm{X}$ and $\mathrm{Y}$ tables (Figure 1) are mounted on one another keeping the 2 -axis profile very low and the system is computer-driven using a programmable motion controller. A datum and an end of travel limit sensor are fitted on each of the tables to trigger the controller when a respective carriage reaches a limit. A perspex sheet was mounted firmly on the 2-axis system in order to accommodate the heating device, which was used to control the temperature of the silicon wafer substrate. A power supply was used to form an electric field between the nozzle and the heating device. Using motion planner software $\mathrm{X}$ and $\mathrm{Y}$ coordinates can be created and downloaded to the 2-axis controller, allowing the 2-axis system to write the path described by the co-ordinates provided.
Printing was carried out under various conditions (see text below) and tracks were sintered by heating to $400^{\circ} \mathrm{C}$ at $2^{\circ} \mathrm{C} \mathrm{min}{ }^{-1}$ and held for $1800 \mathrm{~s}$ before cooling down to the ambient temperature.

\subsection{Microscopical Characterisation}

Samples were coated with carbon before examination by Scanning Electron Microscopy (SEM) and Energy Dispersive X-ray (EDX) analysis. The structure and the surface morphology of the sintered films were investigated using a JEOL JSM-6301F field emission scanning electron microscope operating in the secondary electron mode with an accelerating voltage of $10 \mathrm{kV}$ and with a working distance up to $15 \mathrm{~mm}$. The EDX analysis was performed with an Oxford INCA Energy 200 X-ray energy dispersive spectrometer system.

\subsection{Electrical Testing}

In order to measure the resistance of the tracks, silver (Silver Ink-P6100, Johnson Matthey Catalysts, Enfield, UK) electrodes were placed along the sintered track. Firstly, a multimeter (FLUKE 189 TRUE RMS) was used to measure the resistance of the tracks and later a four-point method was used to obtain a more accurate result by eliminating the resistance of the electrodes and the equipment used.

\section{RESULTS AND DISCUSSION}

When the electric field is applied, the gold suspension jets (Figure 1) and droplets from the jet break-up were deposited on silicon wafers in order to produce a continuous track. The processing parameters such as flow rate, applied voltage and the distance between the substrate and needle exit were varied to find the optimum pattering conditions. Thereafter in all of the patterning experiments the flow rate and applied voltage were set to $5 \times 10^{-11} \mathrm{~m}^{3} \mathrm{~s}^{-1}$ and $1.4 \mathrm{kV}$, respectively, and the distance between the needle exit and substrate was $\sim 0.4 \mathrm{~mm}$. For the layer-by-layer deposition approach, the printing table was moved at $5 \mathrm{~mm} \mathrm{~s}^{-1}$ and a new layer was deposited every 15s. Figure 2 shows an optical micrograph of a single layer track. Due to the well known Marangoni effect, immediately after deposition, most of the particles can be seen clustering together at the edges of the line and a random distribution of particles can be seen at the centre of the track.

Deegan et al [26]., reported that when the contact angle of the droplet on the substrate is $<90^{\circ}$ as in this case, solvent evaporation plays a critical role in drying-mediated self assembly from a dilute colloidal droplet on a wetted surface. In order to observe the gold nanoparticle distribution along the track at different solvent evaporation rates, the substrate was heated at different temperatures $\left(35-85{ }^{0} \mathrm{C}\right)$ before patterning. When 


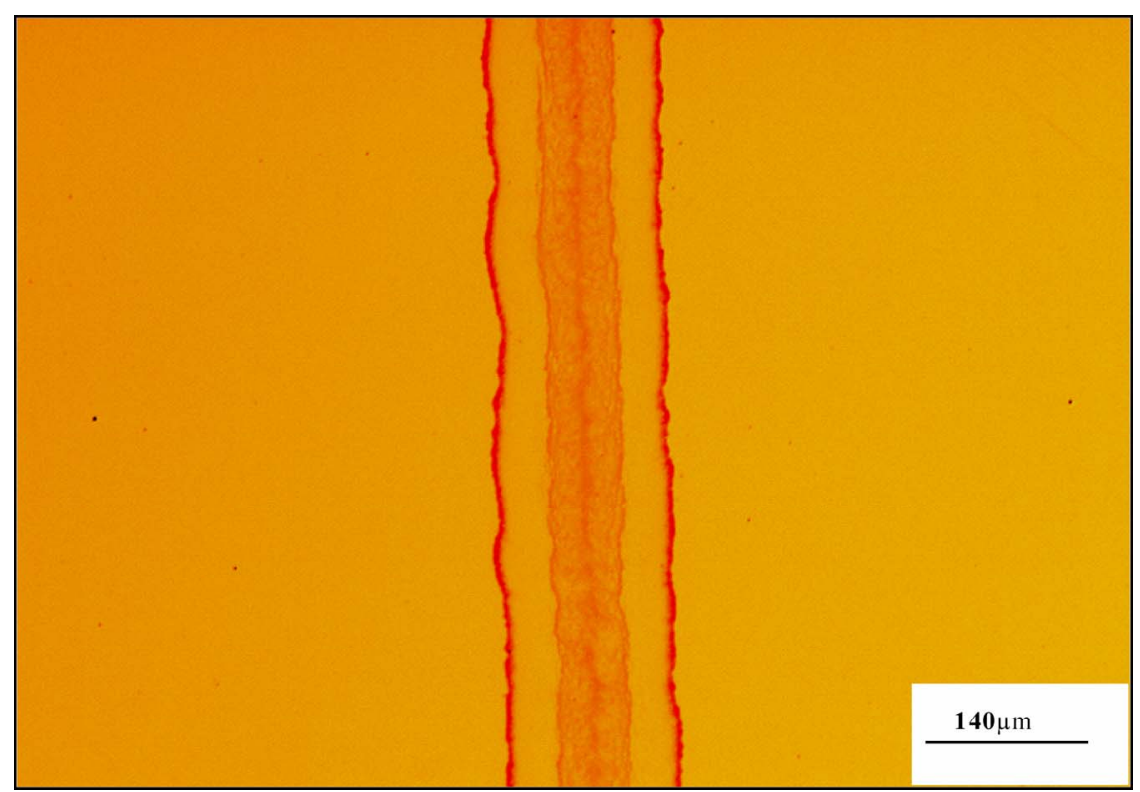

Figure 2. Single layer gold track deposited at $5 \times 10^{-11} \mathrm{~m}^{3} \mathrm{~s}^{-1}$ and $1.4 \mathrm{kV}$.
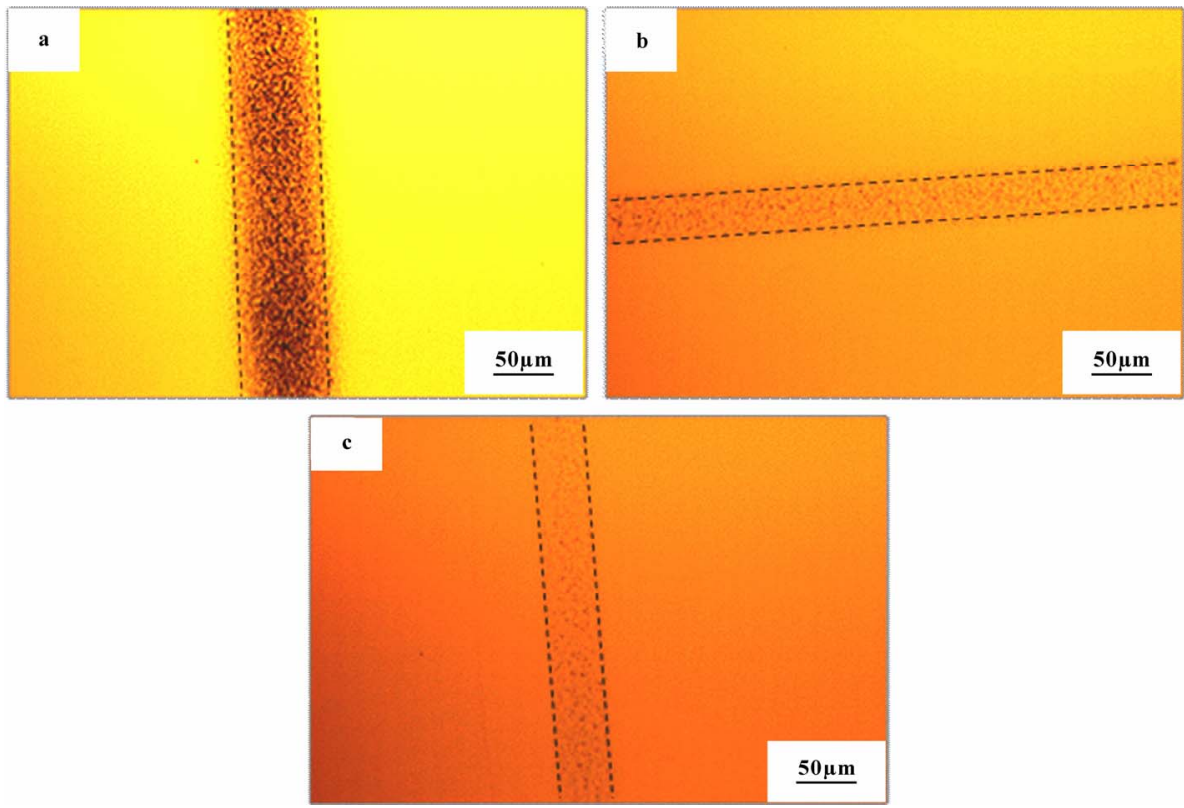

Figure 3. Optical micrographs of the single layer tracks deposited at $85{ }^{0} \mathrm{C}$ with increasing table speeds. a) $3 \mathrm{mms}^{-1}$, b) $6 \mathrm{mms}^{-1}$ and c) $9 \mathrm{mms}^{-1}$. Dotted lines indicates the edge of the track.

the substrate temperature was increased to $>85{ }^{\circ} \mathrm{C}$ patterning was not possible as a stable-jet could not be achieved due to rapid solvent evaporation from the exit of the needle. In order to find out the effect of patterning speed, the table speed was varied from $3-9 \mathrm{~mm} \mathrm{~s}^{-1}$. Figure 3 shows optical micrographs of single layer of track deposited at $85{ }^{\circ} \mathrm{C}$ and with varying table speeds, although a higher speed generates a narrower track, the number of gold particles in the track after a single print- ing pass is higher at the lower speed $\left(3 \mathrm{mms}^{-1}\right)$, therefore, this speed was selected for the multi-layer printing deposition work described below.

Although the particle spreading during solvent evaporation can be reduced significantly by increasing the substrate temperature, due to the low concentration of $\mathrm{Au}$ nanoparticles in the suspension it was not possible to produce dense tracks by a single deposition run, thus a layer-by-layer deposition technique was employed. 


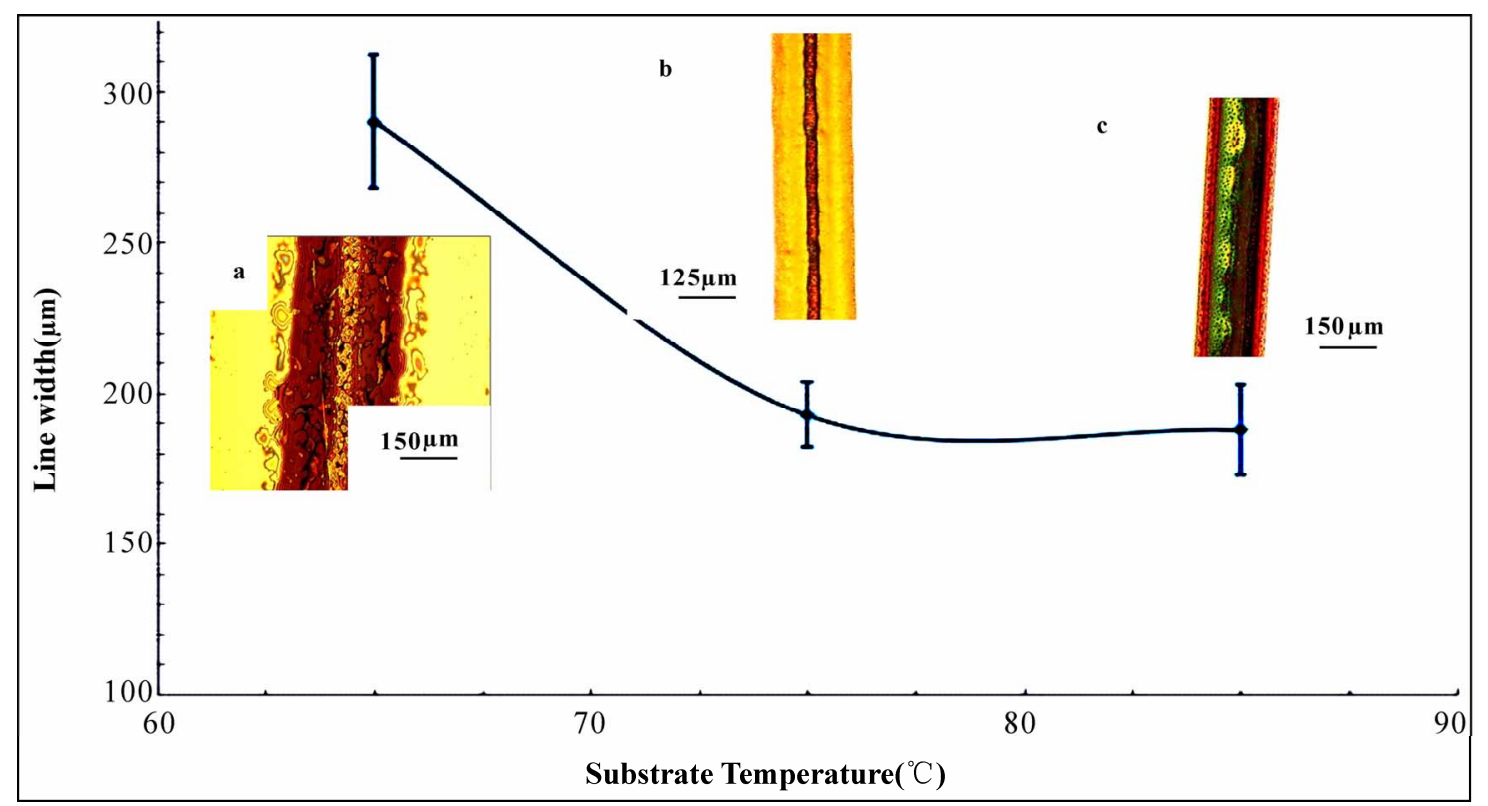

Figure 4. A graph depicting the track width variation with increasing substrate temperature a) $65{ }^{0} \mathrm{C}$, b) $75{ }^{\circ} \mathrm{C}$ and c) $85^{0} \mathrm{C}$. The flow rate was $5 \times 10^{-11} \mathrm{~m}^{3} \mathrm{~s}^{1}$, the applied voltage was $1.4 \mathrm{kV}$ and 50 layers were prepared.

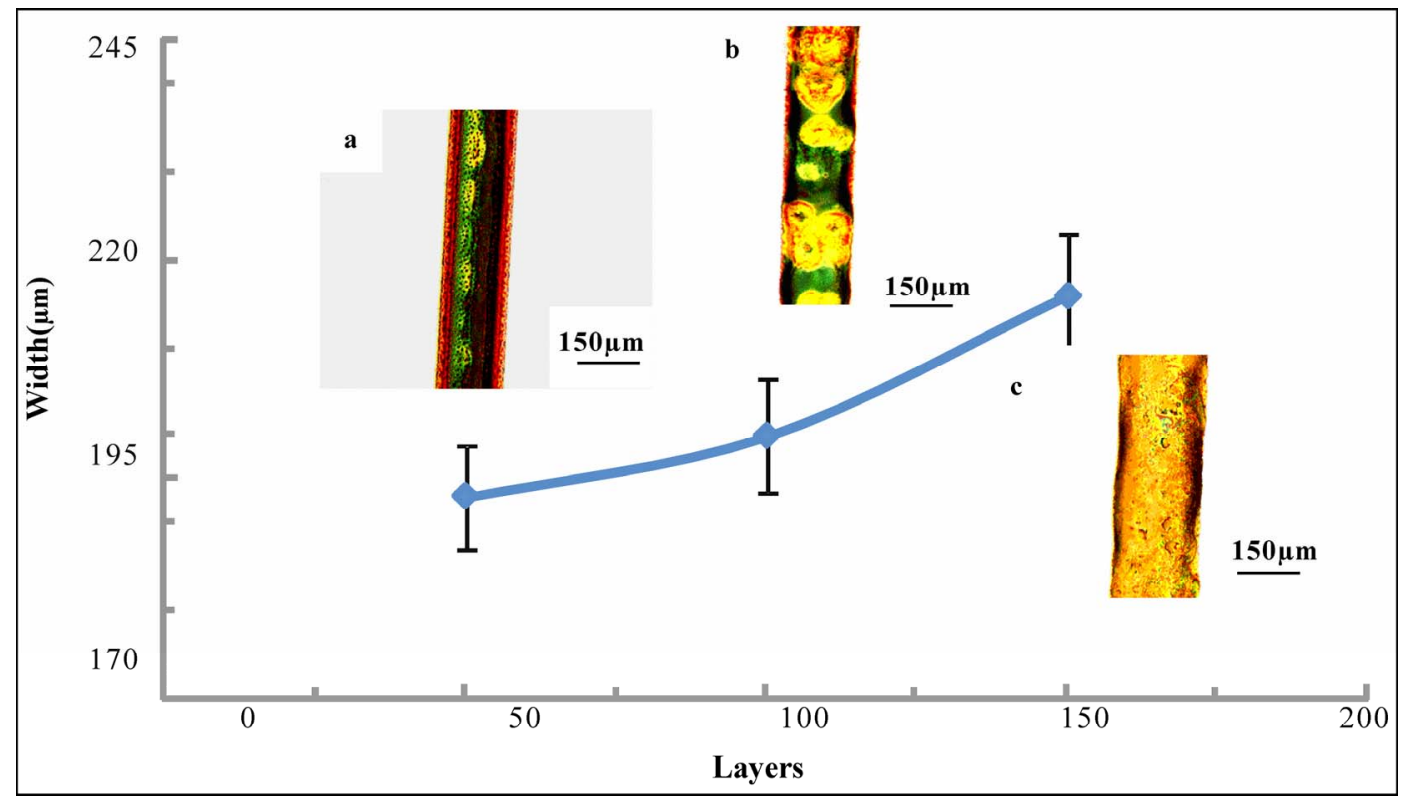

Figure 5. A graph depicting the line width variation with increasing layers at $85^{\circ} \mathrm{C}$, a) 50 , b) 100 and c) 150 . The flow rate was $5 \times 10^{-11} \mathrm{~m}^{3} \mathrm{~s}^{-1}$ and applied voltage was $1.4 \mathrm{kV}$.

Three different substrate temperatures were investigated in conjunction with layer-by-layer deposition, Figure 4 shows the effect of increasing substrate temperature on the track width prepared using 50 layers. The minimum width is achieved at $75^{\circ} \mathrm{C}$. Figure 5 shows the effect on track width due to different numbers of layers deposited at $85{ }^{\circ} \mathrm{C}$. The line width increased with increasing number of layers due to the suttle oscillatory motion of the jet and its digression from the centre line $[23,27]$.
Figure 6 shows a macro image and scanning electron micrographs of a sintered-layered track prepared using a substrate temperature of $85^{\circ} \mathrm{C}$. Although the films appear uniform at a low magnifications, at higher magnifications (Figure 7) they reveal that the films contain "hillocks" (small Au hills that rise above the film). The formation of hillocks is due to preferential landing of some droplets on the substrate and is a characteristic of the fabrication route used and have been explained in 


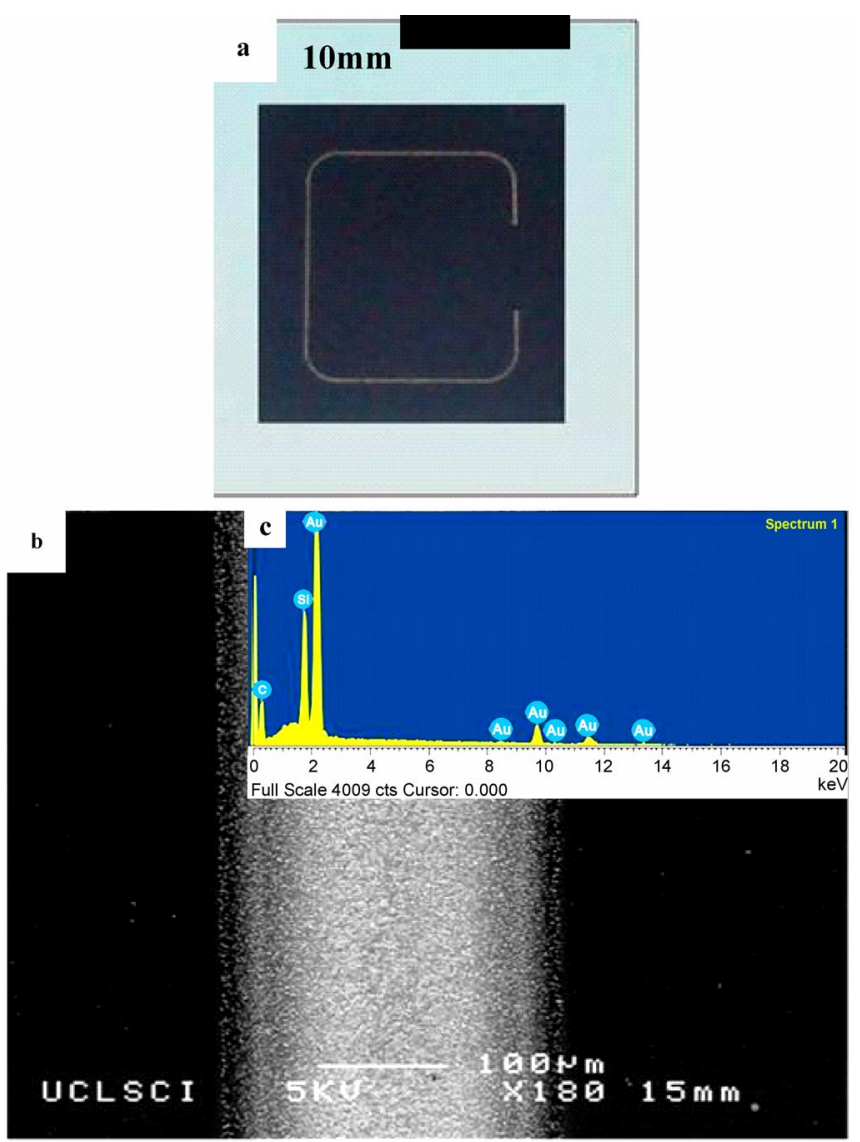

Figure 6. a) Sintered track image, b) scanning electron micrograph of sintered track and c) spectra analysis of (b).
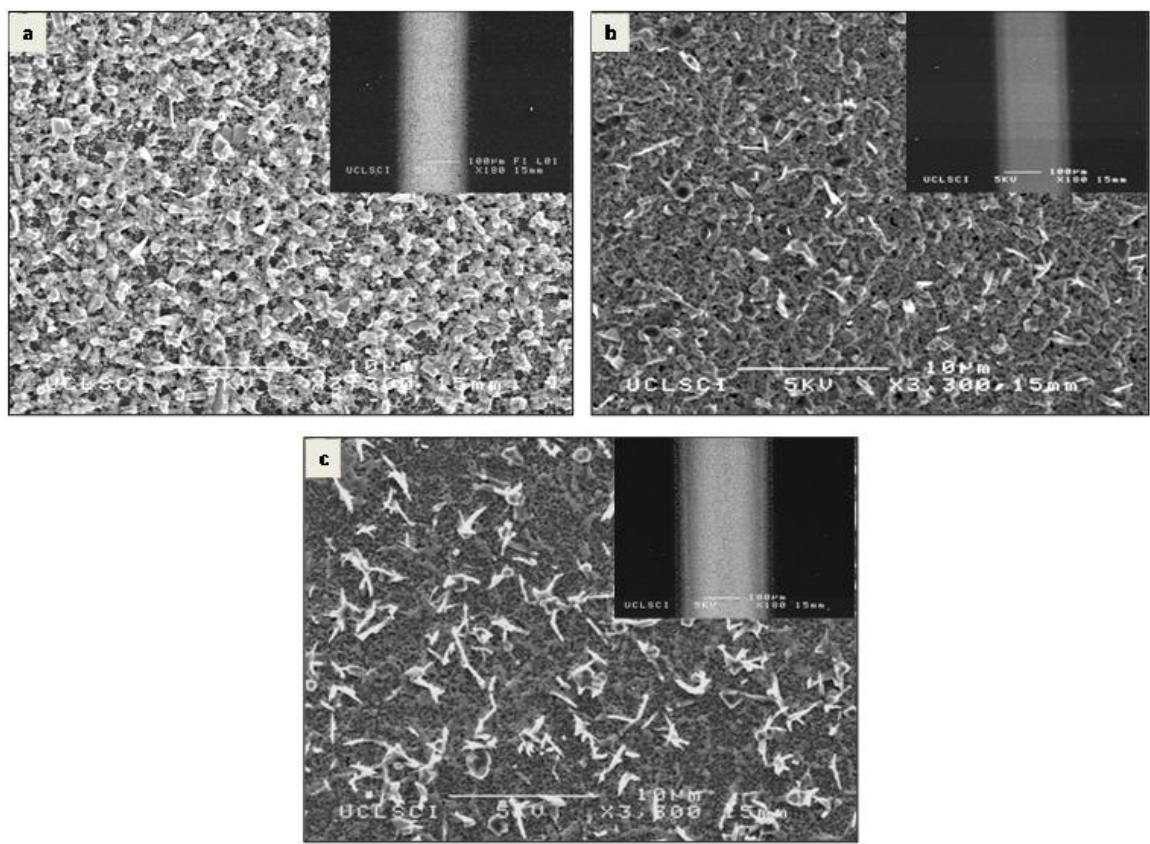

Figure 7. Scanning electron micrographs of the centre of the track with a) 50 layers, b) 100 layers and c) 150 layers. The substrate temperature was set at $85{ }^{\circ} \mathrm{C}$. Bright spots indicate the top of hillocks. 


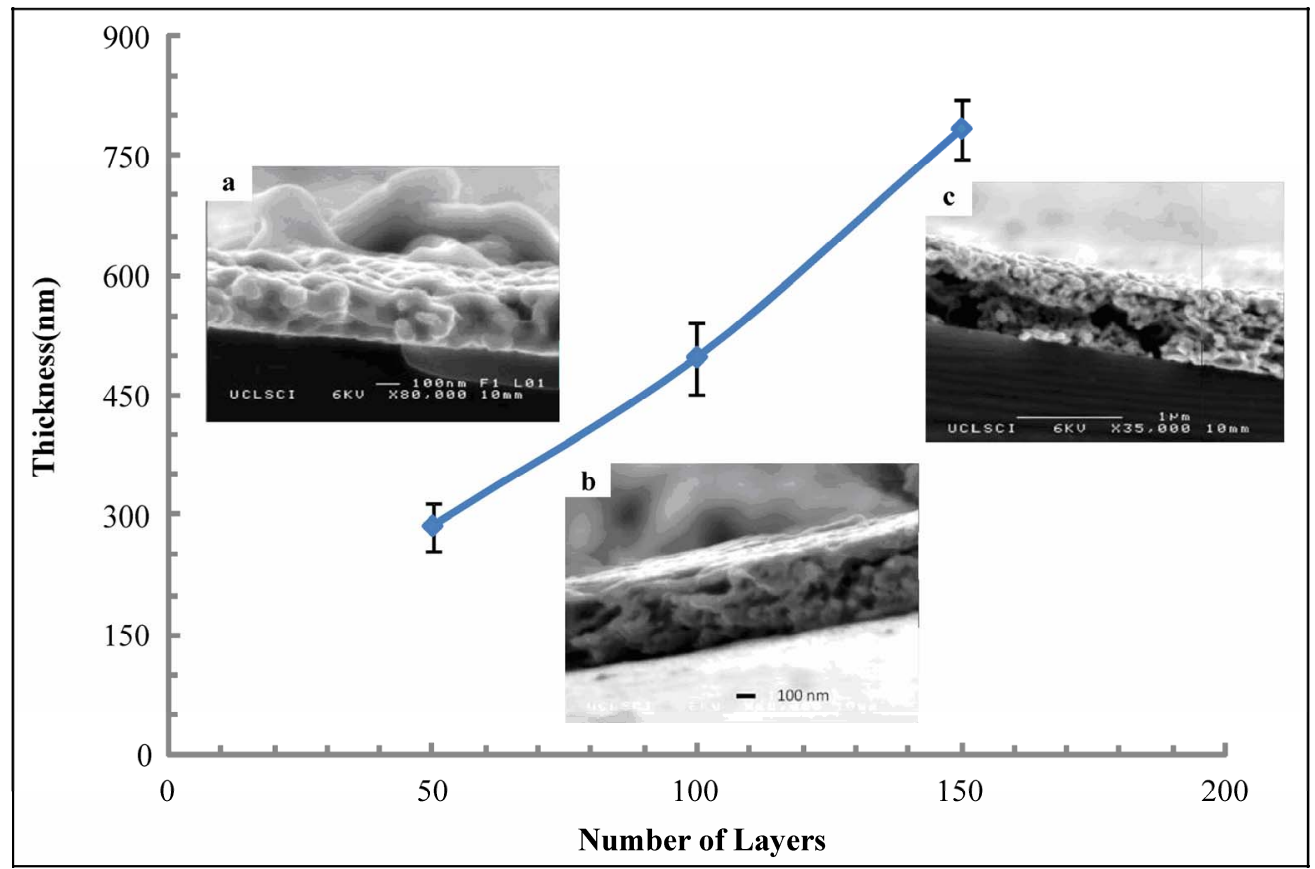

Figure 8. Graph depicting the thickness variation with increasing layers a) 50, b) 100 and c) 150 .

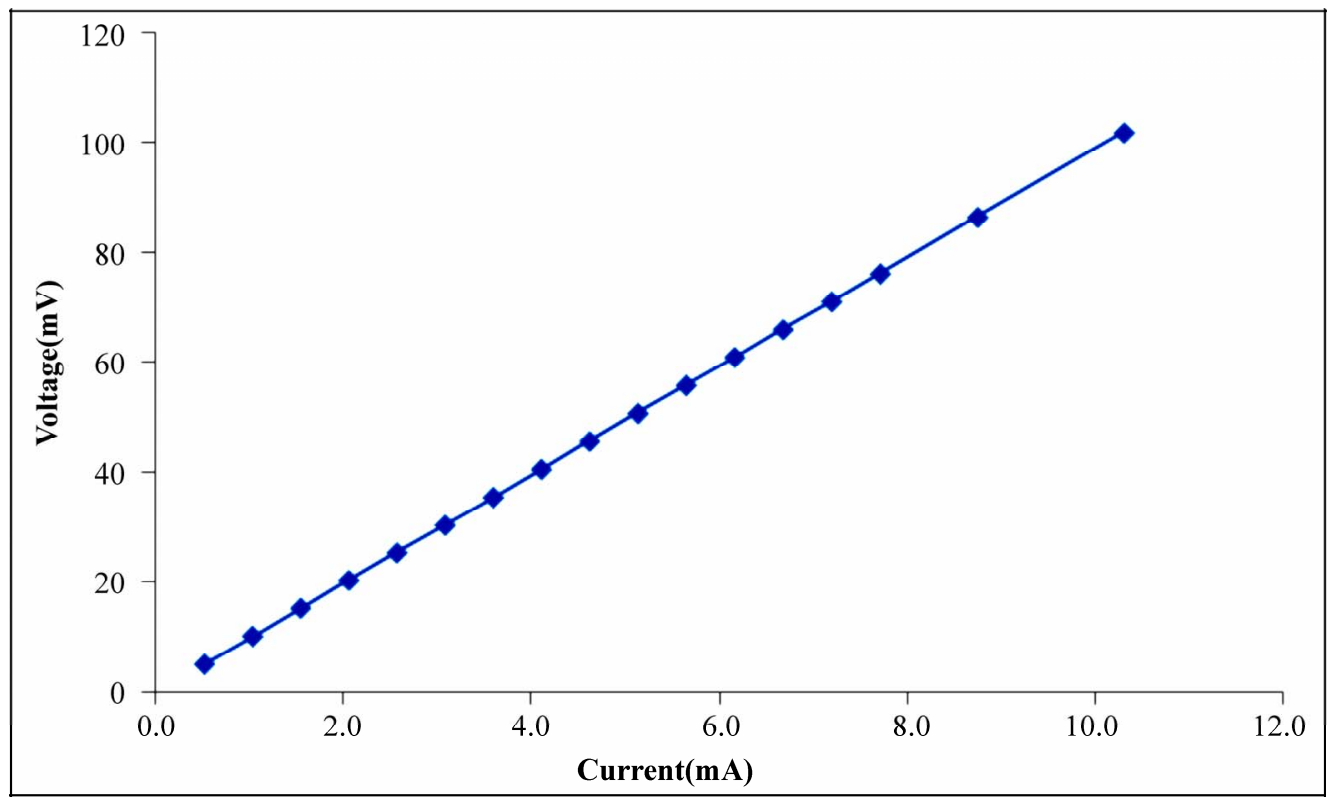

Figure 9. Voltage (V) vs current (I) relationship.

more detail in Samarasinghe et al $[28,29]$. The melting point of $15 \mathrm{~nm}$ diameter gold particles is $\sim 950{ }^{\circ} \mathrm{C}[30,31]$. Therefore, at $400{ }^{\circ} \mathrm{C}$ appreciable sintering and growth of the particles can be expected. Cross-section images of the tracks and the variation of the thickness of the tracks deposited due to increasing the number of layers from 50 to 150 are illustrated in Figure 8. The thickness is an essential parameters for the electrical measurements discussed below.
Figure 9 shows that voltage-current $(V-I)$ characteristics of the tracks showed a linear Ohmic behaviour. The specific electrical resistivity $\rho$ of the produced tracks were calculated by the formula $\rho=R A / L$, where $R$ is the electrical resistance of the line, $L$ is the length of line and $A$ is the cross section area of the line. The resistance of the track was measured using the $V-I$ curve. The cross section area of the track was taken as $A=w t$ where $w$ is the width of the track and $t$ is the thickness of the track. 
Table 2. Resistivity of the printed tracks at different deposition parameters. The resistivity of bulk gold is $2.4 \times 10^{-8} \Omega \mathrm{m}$. Substrate temperature is $85^{\circ} \mathrm{C}$.

\begin{tabular}{cccccc}
\hline Layers & $\begin{array}{c}\text { Length } \\
(\mathbf{m m})\end{array}$ & Width $(\boldsymbol{\mu m})$ & Thickness $(\mathbf{n m})$ & $\begin{array}{c}\text { Resistance } \\
(\boldsymbol{\Omega})\end{array}$ & $\begin{array}{c}\text { Resistivity } \\
(\mathbf{\Omega m})\end{array}$ \\
\hline 50 & 9.5 & 193 & 286 & 47.6 & $2.8 \times 10^{-7}$ \\
100 & 9.5 & 200 & 497 & 24.8 & $2.6 \times 10^{-7}$ \\
150 & 9.5 & 216 & 784 & 9.9 & $1.8 \times 10^{-7}$ \\
\hline
\end{tabular}

The electrical resistivity of the printed tracks (Table 2) using a layer-by-layer approach was in the range of $1.8 \mathrm{x}$ $10^{-7}-2.8 \times 10^{-7} \Omega \mathrm{m}$. Although this value is higher than the resistivity of bulk gold, it is deemed satisfactory especially if one considers the fact that the initial concentration of the gold suspension used in this study was 0.1 wt. $\%$ and no specific processing steps were performed to target the reduction of electrical resistivity. The resistivity compares well with the values of other ink-jet based methods listed in Table 1.

\section{CONCLUSIONS}

This paper demonstrates that gold nanoparticles in dilute suspensions have been successfully assembled to direct write conducting tracks using a simple, economical electric-field assisted printing method. A printing speed of $3 \mathrm{mms}^{-1}$, a suspension flow rate of $5 \times 10^{-11} \mathrm{~m}^{3} \mathrm{~s}^{-1}$, an applied voltage of $1.4 \mathrm{kV}$, and the distance between the needle exit and substrate kept at $0.4 \mathrm{~m}$ were found to be optimum. However, the control of the number of layers deposited and the substrate temperature are crucial parameters to control the track geometry. A track containing of 50 layers deposited with the substrate held at $85^{\circ} \mathrm{C}$ provided a continuous track with a resistivity of $2.8 \times 10^{-7} \Omega$, but this was one order of magnitude above the resistivity of bulk gold.

\section{ACKNOWLEDGEMENTS}

The authors would like to thank the Leverhulme Trust (Grant: F/07 134/BL), EPSRC, UK (platform grant: EP/E045839) for supporting this work. Dr. K.B. Chong at QMUL is thanked for his assistance with electrical measurements; SRS acknowledges the PhD scholarship awarded by UCL, which initiated this work. The help of the Archaeology Department of the University College London is acknowledged for technical assistance with the microscopy.

\section{REFERNCES}

[1] Xu, J., Drelich, J. and Nadgorny, E.M. (2004) La- ser-based patterning of gold nanoparticles into microstructures. Langmuir, 20(4), 1021-1025.

[2] Lee, H.H., Chou, K.S. and Huang, K.C. (2005) Inkjet printing of nanosized silver colloids. Nanotechnology, 16 (10), 2436-2441.

[3] Rogers, J.A., Paul, K.E. and Whitesides, G.M. (1998) Quantifying distortions in soft lithography. Journal of Vacuum Science \& Technology B, 16(1), 88-97.

[4] Chrisey, D.B. (2000) Materials processing - The power of direct writing. Science, 289(5481), 879-881.

[5] Yu, J.H., Kim, S.Y. and Hwang, J. (2007) Effect of viscosity of silver nanoparticle suspension on conductive line patterned by electrohydrodynamic jet printing. $A p$ plied Physics A-Materials Science \& Processing, 89(1), 157-159.

[6] Kamyshny, A., Ben-Moshe, M., Aviezer, S. and Magdassi, S. (2005) Ink-jet printing of metallic nanoparticles and microemulsions. Macromolecular Rapid Communications, 26(4), 281-288.

[7] Chen, D.R., Pui, D.Y.H. and Kaufman, S.L. (1995) Electrospraying of conducting liquids for monodisperse aerosol generation in the $4 \mathrm{Nm}$ to $1.8 \mathrm{Mu}-\mathrm{M}$ diameter range. Journal of Aerosol Science, 26(6), 963-977.

[8] Sullivan, A.C., Scott, K. and Jayasinghe, S.N. (2007) Nanofabrication by electrohydrodynamic jetting of a tailor-made living siloxane sol. Macromolecular Chemistry and Physics, 208, 2032-2038.

[9] Jayasinghe, S.N., Edirisinghe, M.J. and Wang, D.Z. (2004) Controlled deposition of nanoparticle clusters by electrohydrodynamic atomization. Nanotechnology, 15(11), 1519-1523.

[10] Allen, M.L., Aronniemi, M., Mattila, T., Alastalo, A., Ojanpera, K., Suhonen, M. and Seppa, H. (2008) Electrical sintering of nanoparticle structures. Nanotechnology, 19(17), Article Number: 175201.

[11] Kim, D., and Moon, J. (2005) Highly conductive ink jet printed films of nanosilver particles for printable electronics. Electrochemical and Solid State Letters, 8(11), $\mathrm{J} 30-\mathrm{J} 33$.

[12] Szczech, J.B., Megaridis, C.M., Gamota, D.R. and Zhang, J. (2002) Fine-line conductor manufacturing using drop-on-demand PZT printing technology. IEEE Transactions on Electronics Packaging Manufacturing, 25, 26-33.

[13] Kim, D., Jeong, S., Park, B.K. and Moon, J. (2006) Direct writing of silver conductive patterns: Improvement of film morphology and conductance by controlling solvent compositions. Applied Physics Letters, 89(26), article number: 264101.

[14] Dearden, A.L., Smith, P.J., Shin, D.Y., Reis, N., Derby, 
B. and O'Brien, P. (2005) A low curing temperature silver ink for use in ink-jet printing and subsequent production of conductive tracks. Macromolecular Rapid Communications, 26(4), 315-318.

[15] Liu, Z.C., Su, Y. and Varahramyan, K. (2005) Inkjet-printed silver conductors using silver nitrate ink and their electrical contacts with conducting polymers. Thin Solid Films, 478(1-2), 275-279.

[16] Perelaer, J., De Gans, B.J. and Schubert, U.S. (2006) Ink-jet printing and microwave sintering of conductive silver tracks. Advanced Materials, 18(16), 2101-2104.

[17] Bieri, N.R., Chung, J., Haferl, S.E., Poulikakos, D. and Grigoropoulos, C.P. (2003) Microstructuring by printing and laser curing of nanoparticle solutions. Applied Physics Letters, 82(20), 3529-3531.

[18] Bieri, N.R., Chung, J., Poulikakos, D. and Grigoropoulos, C.P. (2004) Manufacturing of nanoscale thickness gold lines by laser curing of a discretely deposited nanoparticle suspension. Superlattices and Microstructures, 35(36), 437-444

[19] Nur, H.M., Song, J.H., Evans, J.R.G. and Edirisinghe, M.J. (2002) Ink-jet printing of gold conductive tracks. Journal of Materials Science-Materials in Electronics, 13, 213-219.

[20] Chung, J.W., Ko, S.W., Bieri, N.R., Grigoropoulos, C.P. and Poulikakos, D. (2004) Conductor microstructures by laser curing of printed gold nanoparticle ink. Applied Physics Letters, 84(5), 801-803.

[21] Park, B.K., Kim, D., Jeong, S., Moon, J. and Kim, J.S. (2007) Direct writing of copper conductive patterns by ink-jet printing. Thin Solid Films, 515(19), 7706-7711.

[22] Lee, D.Y., Hwang, E.S., Yu, T.U., Kim, Y.J. and Hwang, J. (2006) Structuring of micro line conductor using electro-hydrodynamic printing of a silver nanoparticle sus- pension. Applied Physics A-Materials Science \& Processing, 82(4), 671-674.

[23] Lee, D.Y., Shin, Y.S., Park, S.E., Yu, T.U. and Hwang, J. (2007) Electrohydrodynamic printing of silver nanoparticles by using a focused nanocolloid jet. Applied Physics Letters, 90(8), article number: 081905.

[24] Enustun, B.V. and Turkevich, J. (1963) Coagulation of colloidal gold. Journal of the American Chemical Society, 85(21), 3317-3328.

[25] Graf, C., Vossen, D.L.J., Imhof, A. and Van Blaaderen, A. (2003) A general method to coat colloidal particles with silica. Langmuir, 19(17), 6693-6700.

[26] Deegan, R.D., Bakajin, O., Dupont, T.F., Huber, G., Nagel, S.R. and Witten, T.A. (1997) Capillary flow as the cause of ring stains from dried liquid drops. Nature, 389(6653), 827-829.

[27] Reneker, D.H., Yarin, A.L., Fong, H. and Koombhongse, S. (2000) Bending instability of electrically charged liquid jets of polymer solutions in electrospinning. Journal of Applied Physics, 87(9), 4531-4547.

[28] Samarasinghe, S.R., Pastoriza-Santos, I., Edirisinghe, M. J., Reece, M.J. and Liz-Marzan, L.M. (2006) Printing gold nanoparticles with an electrohydrodynamic direct-write device. Gold Bulletin, 39(2), 48-53.

[29] Samarasinghe, S.R., Pastoriza-Santos, I., Edirisinghe, M. J. and Liz-Marzan, L.M. (2008) Fabrication of nanostructured gold films by electrohydrodynamic atomisation. Applied Physics A-Materials Science \& Processing, 91(1), 141-147.

[30] Cortie, M.B. (2004) The weird world of nanoscale gold. Gold Bulletin, 37(1-2), 12-19.

[31] Shim, J.H., Lee, B.J. and Cho, Y.W. (2002) Thermal stability of unsupported gold nanoparticle: a molecular dynamics study. Surface Science, 512 (3), 262-268. 